\title{
TRACES TRAUMATIQUES ET FIGURES PROJECTIVES DES CATASTROPHES DE SYMBOLISATION
}

\author{
Pascal Roman
}

Groupe d'études de psychologie | «Bulletin de psychologie »

2017/4 Numéro 550 | pages 265 à 273

ISSN 0007-4403

Article disponible en ligne à l'adresse :

https://www.cairn.info/revue-bulletin-de-psychologie-2017-4-page-265.htm

\section{Pour citer cet article :}

Pascal Roman, « Traces traumatiques et figures projectives des catastrophes de symbolisation », Bulletin de psychologie 2017/4 (Numéro 550), p. 265-273. DOI 10.3917/bupsy.550.0265

Distribution électronique Cairn.info pour Groupe d'études de psychologie.

(c) Groupe d'études de psychologie. Tous droits réservés pour tous pays.

La reproduction ou représentation de cet article, notamment par photocopie, n'est autorisée que dans les limites des conditions générales d'utilisation du site ou, le cas échéant, des conditions générales de la licence souscrite par votre établissement. Toute autre reproduction ou représentation, en tout ou partie, sous quelque forme et de quelque manière que ce soit, est interdite sauf accord préalable et écrit de l'éditeur, en dehors des cas prévus par la législation en vigueur en France. Il est précisé que son stockage dans une base de données est également interdit. 


\title{
Traces traumatiques et figures projectives des catastrophes de symbolisation
}

\author{
Roman Pascal ${ }^{\mathrm{a}}$
}

\footnotetext{
${ }^{a}$ Laboratoire LARPsyDIS, Institut de psychologie, Faculté des sciences sociales et politiques, Université de Lausanne, Suisse.

Correspondance : Institut de psychologie, Faculté des sciences sociales et politiques, Université de Lausanne, Bâtiment Géopolis 4113, CH-1015 Lausanne, Suisse.

Couriel : pascal.roman@unil.ch
}

Texte reçu le 18 avril 2016 et accepté le 4 juillet 2016

http://www.bulletindepsychologie.net
Résumé : L'auteur reprend, actualise et développe la notion de « catastrophe de symbolisation » dans le cadre des productions aux épreuves projectives. Cette notion avait initialement été proposée dans un article paru en 1997 pour rendre compte des effets de rupture dans les processus de symbolisation. L'hypothèse centrale consiste à considérer que les productions projectives, au Rorschach et au TAT, portent la trace des vécus traumatiques précoces. Ces vécus traumatiques se traduisent par un certain nombre d'expressions projectives (catastrophes de symbolisation) qui témoignent de la mise en échec des processus de symbolisation. Une première discussion du concept de « catastrophe » en mathématique met l'accent sur le double mouvement qui le caractérise : rupture et élaboration d'une nouvelle forme. La définition de trois figures des catastrophes de symbolisation est proposée secondairement, illustrée à partir des protocoles de Rorschach et de TAT de deux adolescents issus d'une recherche consacrée aux adolescents engagés dans des agirs sexuels violents.

Traumatic Traces and Projective Figures of the Disasters of Symbolization

Abstract: The author examines, updates and develops the concept of "disasters of symbolization" in the framework of projective test productions. This notion was first proposed in an article published in 1997 to outline breakage effects in the process of symbolization. The main hypothesis states that early traumatic experiences have an impact on projective productions in the Rorschach test and the TAT. These traumatic experiences are reflected in projective expressions (disasters of symbolization), which testify to the failure of the symbolization process. A first discussion of the concept of a "disaster" in mathematics emphasizes its defining dual components: breakage, and the elaboration of a new form. Secondly, a definition of the three figures of symbolization disasters is provided, and illustrated on the basis of two adolescents' Rorschach and TAT protocols, drawn from a study on adolescents with a history of violent sexual acts. 


\section{INTRODUCTION}

Il y a près de vingt ans, je publiais un article dans la revue Psychologie clinique et projective, dans le cadre d'un volume consacré à la psychosomatique intitulé : "Troubles somatiques et catastrophes de symbolisation »(Roman, 1997a). Cet article fait partie des textes régulièrement cités par les jeunes collègues, en particulier comme soutien méthodologique dans les démarches projectives en lien avec les diverses problématiques, au sein desquelles la question du traumatisme se trouve sur le devant de la scène, tout comme l'un des chapitres publié la même année, intitulé "La méthode projective comme dispositif à symboliser » (Roman, 1997b).

À partir de là, on peut s'interroger, à rebours, sur ce qui fonde un intérêt particulier pour ces publications, ainsi que sur l'actualité, la pertinence et le sens des propositions qu'elles contiennent. Si l'on porte un regard après-coup, la mise en perspective de ces deux publications préfigure le projet dans lequel se sont inscrits mes travaux de recherche depuis une vingtaine d'années, dans la double lignée des pensées de Chabert, d'une part, et de Roussillon, d'autre part. En effet, je défendais respectivement, dans ces deux textes :

- au plan d'une théorie de la méthode, l'affirmation de la fonction symbolisante des épreuves projectives ; l'épreuve projective considérée comme « dispositif à symboliser » (Roman, 1997b), avec une déclinaison qui s'étire de l'offre à symboliser à la contrainte à symboliser, dans la perspective d'épreuves qui invitent à repasser sur les traces de l'histoire des processus de symbolisation, et qui soutiennent un « processus psychanalytique bref » (Brelet, 1986) ;

- au plan clinique et méthodologique, la mise en évidence de l'intérêt du repérage, à partir d'une clinique psychosomatique de l'enfant, de la trace des écueils du travail de symbolisation dans les protocoles d'épreuves projectives, à partir de l'identification d'un certain nombre de manifestations projectives, que j'ai nommées alors « catastrophes de symbolisation ».

Il s'agit ici, tout spécialement de tenter de mettre en perspective les propositions liées à la définition et à l'illustration des catastrophes de symbolisation, au regard de l'actualité de ma recherche clinique, largement marquée par l'exploration de la problématique du traumatisme, car, au fond, ce qui sous-tend la perspective des catastrophes de symbolisation, c'est bien la mise en évidence, dans la rencontre projective, de ce qui fait trace du traumatisme, en tant que le traumatisme (c'est-à-dire le vécu traumatique, du point de vue de la subjectivité et de son atteinte) renvoie à une mise en échec des processus de symbolisation.

On peut rappeler que la définition du traumatisme, dans son acception psychanalytique, comporte deux aspects :

$1^{\circ}$ le traumatisme psychique requiert deux ingrédients : l'expérience traumatique, caractérisée par un trop-plein d'excitation, que la vie psychique du sujet ne peut pas traiter, et le sentiment d'une absence de recours ; en d'autres termes, le traumatisme consiste dans une double expérience : une situation qui fait violence à la vie psychique, comme des mauvais traitements, des violences physiques ou sexuelles, des négligences, des inadéquations, des absences ou des abandons, ce que Janin (1996) nomme «noyau chaud du traumatisme », et l'expérience de ne pas avoir pu compter, dans son environnement, sur un adulte secourable ; selon la formulation de Winnicott (1989) : « là où quelque chose aurait du se passer pour l'enfant, c'est le rien dont il a fait l'expérience », situation qui fait éprouver, sans pouvoir le traduire dans le langage, une « crainte de l'effondrement», ce que Janin (1996) nomme «noyau froid du traumatisme »;

$2^{\circ}$ le traumatisme psychique, pour se constituer dans sa dimension délétère, se constitue à partir de deux temps : le premier temps, celui du vécu traumatique à proprement parler, situé dans la période de l'enfance et bien souvent maintenu dans un lieu hors-psyché (par l'intervention du clivage), et le second temps (l'après-coup), situé à l'adolescence ou à l'âge adulte, temps de la sexualisation du traumatisme, temps où le traumatisme prend sens dans l'économie psychique génitale.

Voici donc ce que j'écrivais dans la revue Psychologie clinique et projective : « En d'autres termes, on peut se demander comment le déficit de symbolisation inscrit le corps comme scène du symptôme (dans un surinvestissement du sensoriel, dans une mise en absence impossible de la sensorialité) ou comment le déficit somatique réactive, pour les mettre en question, les structures mêmes de la symbolisation. » (Roman, 1997a, p. 77).

Ce que je me proposais d'interroger alors, dans le contexte des affections psychosomatiques chez l'enfant, concerne la singularité du destin pulsionnel dans ces configurations cliniques, destin inscrit dans la dialectique complexe entre corps et psyché. Il importe par ailleurs de signaler que ce qui constitue aujourd'hui une part importante de mes investissements de recherche concerne la singularité du destin pulsionnel dans les agirs sexuels violents au temps 
de l'adolescence (Roman, 2012, 2014a, 2015a). L'axe de mon travail consiste à considérer que la mise en jeu du corps de l'adolescent dans le corpsà-corps imposé à la victime peut être conçue comme l'ouverture d'une nouvelle scène. Cette nouvelle scène, inscrite dans un processus de répétition, va tenter de mettre en sens des éléments de l'histoire traumatique, dans le prolongement de l'hypothèse selon laquelle l'agir peut être conçu comme avatar et comme relance du travail de symbolisation.

C'est donc dans le fil de ces intérêts cliniques autour de l'adolescence, des remaniements qui la caractérisent et des catastrophes auxquelles elle nous confronte, que je me propose de ré-évaluer l'efficience de la notion de catastrophe de symbolisation.

\section{VERS UNE DÉFINITION DES CATASTROPHES DE SYMBOLISATION}

Mais que sont ces catastrophes de symbolisation ? Si le terme de «symbolisation » fait partie de notre patrimoine conceptuel dans le champ de la psychanalyse, avec en particulier les propositions de distinction entre symbolisation primaire et secondaire (Roussillon, 1991, 2014) le terme de « catastrophe » ne va pas de soi.

La référence que je propose ici du terme de catastrophe déborde bien sûr le sens commun du terme. Celui-ci, issu du grec, renvoie à l'idée d'un bouleversement et de dénouement (même si la dualité sémantique du terme de dénouement peut se rapporter à une conception clinique de la catastrophe : défaire les nœuds et trouver une issue). Il faut noter, d'ailleurs, que la connotation péjorative du terme de catastrophe (associé à la destruction) n'est pas nécessairement avérée (d'un point de vue étymologique, la catastrophe, c'est également un coup de théâtre), même si cette connotation péjorative domine actuellement les usages de ce terme en français.

D'un autre côté, le terme de catastrophe se trouve, depuis les travaux du célèbre mathématicien Thom sur la théorie des catastrophes, teinté d'une autre dimension. Il a acquis un versant éminemment dynamique, ainsi que le souligne Virole (1992) : « La théorie des catastrophes de René Thom propose "une épistémologie du regard", selon la belle expression de Krzystof Pomian, posée sur les formes et pouvant à partir d'elles remonter jusqu'à la dynamique dont elles sont issues par déploiement. » (Virole, 1992, p. 31). La théorie des catastrophes cherche ainsi à saisir et à modéliser, au plan mathématique, un certain nombre de changements, ainsi que les traces de ces changements. Je sollicite, à nouveau, les propositions de Virole, dans sa fonction de passeur entre le monde des mathématiques et le monde de la psychologie, énoncées dans le prolongement de l'article de 1992 : «Selon ce deuxième sens (il faut entendre ici : le sens de la théorie des catastrophes), une catastrophe est la disparition d'une forme stable. Cette disparition aboutit à l'établissement d'une nouvelle forme consécutivement à une modification des forces agissantes sur le système » (voir http://www.benoitvirole.com/).

C'est bien dans le fil de cette acception qu'il nous faut alors entendre le terme de catastrophe de symbolisation : comme une modalité qui témoigne d'une mise en tension des processus et qui concourt au travail de symbolisation dans la double valence désorganisation / réorganisation. Dit autrement, les catastrophes de symbolisation rendent compte d'un coup de théâtre au sein de la vie psychique, dont la scénarisation sur la scène projective, que l'on peut penser comme une nouvelle scène du traumatisme, informe de ses effets sur la dynamique de la vie psychique.

Dans l'article sur les catastrophes de symbolisation (Roman, 1997a), je ne propose pas, à proprement parler, de définition des catastrophes de symbolisation, mais j'en décris quatre formes d'expressions principales (que je développe comme « quatre indices »), illustrées par des fragments de réponses au Rorschach. Ces quatre formes expressives portent sur les réponses dans la dynamique de leur production ; il s'agit, en effet, au-delà du contenu des réponses, considérées à partir de leur cotation, de rendre compte du contexte de leur production. Voici comment se déclinent alors les catastrophes de symbolisation :

- un primat de la perception immédiate ou réponses de l'ordre du vu-vécu ;

— des effets de blanc affectant les liaisons au sein du discours ;

— des îlots de déni, témoins de ruptures dans le processus représentatif ;

- une effraction des fonctions contenantes et pare-excitatrices dans le jeu transférentiel.

Comme je l'ai déjà précisé, ces différentes formes expressives constituent autant de traces des écueils du travail de symbolisation. Je propose aujourd'hui de les reformuler autour de trois axes, qui méritent d'ailleurs d'être abordés tout autant à partir du Rorschach que des épreuves thématiques (CAT, TAT) ou des épreuves de jeu (Scéno-test en particulier) :

- l'axe de l'investissement sensoriel du matériel projectif dans la production de la réponse (symé- 
trie, couleur et estompage...), ou axe de l'élaboration de l'affect; l'enjeu sous-jacent est celui de l'advenu de l'affect ;

- l'axe de la continuité du processus représentatif dans l'élaboration du discours, regroupant effets de blanc et îlots de déni (qui renvoient, de fait, à la même logique processuelle) ou axe de la construction $d u$ fond de continuité narcissique ; l'enjeu sous-jacent est celui de la construction de la scène, en tant que fonction-support ;

- l'axe de l'engagement transférentiel, témoin de la qualité des pare-excitations, ou axe du traitement de l'excitation dans le lien ; l'enjeu sousjacent est celui de la plasticité opposée à la porosité des limites, avec le repérage des agirs de transfert qui en constituent des indicateurs particulièrement pertinents (Freud, 1915) ${ }^{1}$.

\section{PSYCHODYNAMIQUE DES VIOLENCES SEXUELLES À L'ADOLESCENCE ET SCÈNE PROJECTIVE}

La présentation de quelques éléments théorico-cliniques à partir desquels pourra être appréhendée, dans une approche psychodynamique, la clinique projective des adolescents engagés dans des agirs sexuels violents, du point de vue des catastrophes de symbolisation, permettra de situer le contexte de mise à l'épreuve de la problématique du traumatisme.

J'ai soutenu, depuis plusieurs années, dans la suite des travaux de Balier (1996) et Ciavaldini (1999), en particulier, l'hypothèse selon laquelle les agirs sexuels violents à l'adolescence contribuent au processus adolescent. Si ces agirs signalent une souffrance au lieu du destin du sexuel infantile dans le processus adolescent (Gutton, 1991, 1996), ils constituent également une opportunité pour une relance de ce processus. À partir de là, on peut comprendre que l'agir commis dans le temps de

1. Bokanowski (http://www.spp.asso.fr/wp/?p=5853) cite les propos de Freud (1915) dans son texte « Remarques sur l'amour de transfert» et évoque un « transfert agi (ou un « agir de transfert»), dans lequel l'acte vient remplacer la parole, vient interrompre le jeu transférentiel » : «Il y a un changement complet de la scène, comme si une pièce avait cédé la place à une réalité effective faisant subitement irruption, un peu comme quand retentit le signal de l'incendie pendant une représentation théâtrale. » (Freud, 1915, p. 202). On peut noter que plus loin Freud complète : «La maladie aurait atteint ce vers quoi tendent tous les malades dans l'analyse : agir quelque chose, répéter dans la vie ce qu'elle doit seulement remémorer, reproduire en tant que matériel psychique et maintenir dans le domaine psychique. » (Freud, 1915, p. 206). l'adolescence autorise l'ouverture d'une autre scène pour les vécus traumatiques infantiles, remobilisés au travers du traumatisme pubertaire, si l'on veut bien accepter de penser la quête de sens contenue dans l'agir violent adolescent.

Si l'on suit les propositions de Ciavaldini (2005), qui qualifie l'agir comme un «affect inachevé », on peut alors penser que l'émergence ou le primat de la sensorialité (ou de la quête de sensorialité) contenue dans l'agir sexuel violent témoignerait dans l'actuel de l'inachèvement de l'affect. Ravit, Di Rocco, Bécache, Carka (2013) soulignent, par ailleurs, que se trouve mobilisé, dans l'agir, une « quête de la part non advenue de la position subjective » et la quête des échos de «ce qui s'est perdu dans le reflet du miroir primaire ». Le sensoriel est mobilisé à cet endroit, comme la voie (une voie) pour renouer le fil d'un processus de symbolisation, toujours, répétitivement, en échec. Dans ce contexte, la « surenchère sensorielle » (Ravit, 2010) pourrait être comprise comme étant au service d'une tentative de mise en figuration du traumatique.

Cette figuration nécessite une scène, une autre scène, qui est tout à la fois la scène de l'agir sexuel violent, en ce qu'il témoigne d'une mise en jeu de l'affect sur le fond d'une méconnaissance de ses propres affects et des affects de l'autre (la victime), et la scène de la rencontre clinique en appui sur le dispositif projectif. En effet, la scène projective peut être conçue comme scène de répétition traumatique, à la fois, scène de la catastrophe et scène des coups de théâtre. L'excitation portée par le donné à voir du matériel projectif (Roman, 1998) met à l'épreuve les potentiels de symbolisation et invite l'adolescent à parcourir les traces de l'infantile dans ses inscriptions traumatiques, avec, en particulier, la perspective du traitement de l'auto-séduction propre au temps pubertaire (Gutton, 1991), et la remise en jeu de la subversion libidinale dans l'investissement de la nouvelle sexualisation du corps (Dejours, 2013).

\section{CLINIQUE PROJECTIVE DES CATASTROPHES DE SYMBOLISATION}

La présentation des formes expressives des catastrophes de symbolisation se déclinera selon les trois axes précédemment définis, en appui sur les protocoles d'épreuves projectives d'adolescents mis en cause et condamnés pour des actes d'ordre sexuel, recueillis dans le cadre de la recherche EvAdoGroupe ${ }^{2}$. Cette recherche est consacrée à l'éva-

2. La recherche EvAdoGroupe (évaluation des processus de changements des adolescents engagés dans des agirs 
luation des potentiels de changement des adolescents auteurs de violence sexuelle, en appui sur un protocole de recherche longitudinal, qui comporte, entre autres, une proposition de test-retest avec les épreuves du Rorschach et du TAT. J'ai choisi, de manière un peu aléatoire, les protocoles de Rorschach et de TAT de deux adolescents pour illustrer la clinique projective des catastrophes de symbolisation : Corneille, âgé de 18 ans, et Charles, âgé de 17 ans. Les références aux protocoles respectifs de Rorschach et de TAT des deux adolescents seront identifiées de la manière suivante, pour chacun des trois axes : Rorschach Corneille : A ; TAT Corneille : B ; Rorschach Charles : C ; TAT Charles : D.

Axe de l'investissement sensoriel du matériel projectif dans la production de la réponse (traces quasi-hallucinatoires, retour du vu ou de l'entendu, sensations hallucinées), ou axe de l'élaboration de l'affect

Un certain nombre de figures des catastrophes de symbolisation, témoins du primat de l'investissement perceptif-sensoriel, peuvent être identifiées à partir des protocoles d'épreuves projectives des deux adolescents.

\section{A - Corneille (épreuve de Rorschach)}

- L'accrochage à la symétrie (qui apparaît présent de manière récurrente sur l'ensemble du protocole) que l'on peut envisager dans une fonction d'auto-soutien subjectif (on pourrait évoquer la notion de « vertébration subjective ») :

I : Faut que je raconte quoi ?... un bassin et l'os... $\mathrm{Y}$ a toujours un axe de symétrie au milieu, faudrait un miroir, ça sera sur toutes les images j'en suis sûr. On peut s'imaginer beaucoup de choses là-dessus mmmh.

- Cette fonction d'auto-soutien subjectif se déploie dans le contexte d'une mise en jeu, par l'adolescent, de l'investissement du narcissisme :

VII : (prend la planche à la main) Bon faut que je répète axe de symétrie. Je crois que toutes je les ai vues, pourrait faire office de deux silhouettes, deux têtes, l'air d'être deux filles quoique, ou même pas... Elles se regardent l'une à l'autre ou dans le miroir avec l'axe de symétrie je regarde ce que je suis.

sexuels violents pris en charge dans des groupes thérapeutiques) est dirigée par le professeur Roman dans le cadre du LARPsyDIS, Institut de psychologie, à l'université de Lausanne (Suisse). Cette recherche est soutenue par le Service de protection de la jeunesse (SPJ - État de Vaud, Suisse) et le Centre ressources pour les intervenants auprès des auteurs de violences sexuelles (CRIAVS - Rhône-Alpes, France).
On peut s'interroger, à partir de cette réponse, sur la tentative de Corneille d'ouvrir et de créer un espace dans l'intrapsychique et dans l'intersubjectivité, pour la composition de l'affect (Roussillon, 2002).

- L'accent sur la couleur dans les planches pastel, au travers de réponses, dans lesquelles la couleur prime sur l'élaboration de la forme ; en filigrane, émerge une interpellation sur l'origine et la matière du matériel, interpellation qui contribue à soutenir l'émergence de l'affect :

VIII : Bon toujours axe de symétrie... Pourrait faire office ces deux taches rouge sur les côtés d'insectes, des lézards, y a quand même des pattes, des insectes, machin comme ça. En tout cas ça a été fait avec de l'aquarelle, on voit tout de suite, tous je pense.

IX : De nouveau présence de couleurs et axe de symétrie. Plutôt ça ressemble à deux dragons ou deux hippocampes. Présence orange pour le feu, aussi du blanc, du rouge, du jaune. Mélange de couleurs chaudes. Si on veut du plastique ou de l'aluminium, plus une flamme verte, verte, bleue, violet enfin bref quelque chose qu'on voit que c'est toxique (il regarde par la fenêtre).

On peut noter, en particulier pour la planche IX, l'évolution du sensoriel visuel (les couleurs nommées) et cénesthésique (la chaleur, avec « couleurs chaudes ») en direction de l'affect (la référence au «toxique ») qui prend appui sur l'identification de la matière (« du plastique, de l'aluminium »).

\section{B - Corneille (épreuve du TAT)}

- La confrontation avec l'une des planches les moins figuratives du TAT (planche 11) mobilise une tentative de construire le stimulus à partir de l'explicitation de la saisie perceptive-sensorielle :

11 (prend la planche à la main) : Ça ça me rappelle quelque chose cette image, je suis sûr de l'avoir vue. Bon ben là on est dehors, au bord peut-être d'un château, y a des chutes de pierres, un stock de pierres, une ouverture, une forteresse, en tout cas un mur.

On notera l'importance donnée aux aspects liés à la construction des espaces (dedans-dehors, passage, délimitation...), sur le fond d'un rappel dont on peut relever l'hypothèse d'une émergence quasi-hallucinatoire ( Ça ça me rappelle quelque chose cette image, je suis sûr de l'avoir vue »).

\section{C- Charles (épreuve de Rorschach)}

- Le protocole de Charles est, quant à lui, émaillé de réponses qui témoignent de la trace 
perceptive brute issue de l'émergence pulsionnelle, sur le fond d'une persévération, centrée sur des réponses d'armes, comme vecteur d'une pulsionnalité violente :

II : un impact de balle. Autre chose ? Non ? on pourrait croire à un impact de balle comme ce trait au milieu. C'est tout.

VI : ? on pourrait croire à un impact de balle comme ce trait au milieu. C'est tout. (enquête : l'impact de balle, l'entrée, la sortie et le trajet de la balle).

Axe de la continuité du processus représentatif dans l'élaboration du discours, regroupant effets de blanc et îlots de déni, ou axe de la construction du fond de continuité narcissique

Les protocoles des deux adolescents retenus pour ces illustrations offrent l'opportunité de saisir des figures des catastrophes de symbolisation marquées par les ruptures dans le processus représentatif.

\section{A - Corneille (épreuve de Rorschach)}

- La construction du fond, en appui sur la symétrie, met à mal (écrase ?) le processus de figuration :

I (prend la planche à la main) : Faut que je raconte quoi ? ... un bassin et l'os ... Y a toujours un axe de symétrie au milieu, faudrait un miroir, ça sera sur toutes les images j'en suis sûr. On peut s'imaginer beaucoup de choses là-dessus mmmh.

La reprise de la verbalisation face à cette première planche s'impose, ici pour rendre compte sur les effets de rupture (effets de banc, îlot de déni). On peut en effet relever le contraste entre l'annonce de la potentialité imaginaire de la planche ( «On peut s'imaginer beaucoup de choses là-dessus »), et l'impossibilité pour l'adolescent de produire des réponses supplémentaires à cette planche, au-delà de la réponse anatomique et quasi-dévitalisée élaborée au préalable (« un bassin et l'os »).

- La référence au blanc semble atteindre la continuité de la représentation :

II : ça pourrait éventuellement ressembler cet espace blanc, je sais pas si vous vous représentez la Tour Mallaig, enfin il manque la tour principale. Je peux pas dire quelque chose de plus.

$\mathrm{Au}$ travers de cette réponse à la planche II, dont on peut souligner la participation déterminante du détail blanc central, l'impact de cette vacance apparaît double, du point de vue de la continuité du processus représentatif de Corneille : d'une part limitation (voire censure) de la représentation ( la
Tour Mallaig, enfin il manque la tour principale ») et d'autre part limitation de l'engagement projectif (« je peux pas dire »).

- L'attaque et/ou la disqualification de la représentation humaine se développe en lien avec la référence à la caricature :

III : Axe de symétrie. Ça ressemble à deux espèces d'êtres humains, caricaturés, en tout cas tache rouge au centre ressemble à un nœud papillon... on peut tourner en rond.

III' - retest : Toujours axe de symétrie. Ça pourrait être un peu un nœud papillon là au milieu, ça qui est gris fait office de deux silhouettes, c'est un peu imaginaire, c'est pas deux hommes. C'est un peu ces arts chinois tous ces machins. Ça ressemble à rien. On peut bien se demander à quoi ça sert d'accrocher ça chez soi.

On peut mesurer la place et la fonction de l'investissement de la sensorialité, tout à la fois point d'ancrage ou de réassurance dans le processus représentatif, et témoin du risque d'une disqualification de l'imaginaire. Par ailleurs, lors du retest, l'interpellation sur l'intérêt d'une exposition de la planche (« à quoi ça sert d'accrocher ça chez soi ») interroge une autre forme de rupture : quelle est la compréhension de l'adolescent quant au sens de la passation de l'épreuve projective ? Quelle projection peut être identifiée dans cet appel au psychologue quant à un accrochage chez soi de la planche ? De quel risque témoigne la rencontre de la planche du point de vue de l'intime?

- L'insistance sur la structure du stimulus vient prendre le pas sur la représentation :

IV : Axe de symétrie. On pourrait presque dire que c'est un géant ou quelque chose comme ça avec ses deux grands pieds en bas de l'image (il se gratte la tête)... crachant quelque chose au centre, cet espèce de couloir de l'image, de haut en bas, verticalement.

La référence au «couloir de l'image » et à la vectorisation (« de haut en bas, verticalement ») tend à submerger le potentiel de mise en image face au stimulus.

- Le trop peu de représentation ou le trop du stimulus fait vivre le risque de la discontinuité et mobilise un mouvement actif face à la planche :

VI : une seule chose mais qui représente même pas toute l'image ... je voudrais retoucher un peu ... un volcan, la cheminée au centre, juste la cheminée, un couloir d'une cheminée. Autour c'est déjà trop, faut que ce soit juste précis. 
Face à l'intensité de l'excitation mobilisée par le stimulus, et à l'expression du sentiment d'un débordement qui ouvre sur une limitation de la potentialité représentative («juste la cheminée »), Corneille tente de reprendre le contrôle sur le stimulus même («je voudrais retoucher un peu »).

\section{B - Corneille (épreuve de TAT)}

- La quête du support pour la représentation confronte l'adolescent au risque de la construction de la forme sur le fond, et ouvre sur une confusion entre forme et fond :

1 : C'est quoi ça ? (tourne la planche) c'est quoi ce machin ... c'est quoi ça ... un support pour un livre ? Un livre lui-même ? Un tableau ? ... je vois un homme, enfin un homme plutôt un garçon qui est en train de regarder cet objet là mais il est à moitié endormi ... Qu'est-ce que vous voulez que j'ajoute ? C'est peut-être une table vu qu'il est assis ... non quoi il est assis.

La confusion entre la représentation et son support (« ... un support pour un livre ? Un livre luimême ? »), considérés de manière interchangeable, témoigne de la fragilité des assises narcissiques de base ; l'inférence qui concourt à la représentation de l'objet («C'est peut-être une table vu qu'il est assis ») interroge, d'une autre manière, la précarité de l'instance représentative, sur le fond d'une mise à l'épreuve de la représentation de l'absence de représentation.

16 : Vraie-fausse ? Il peut y avoir deux histoires : une je ne dis rien c'est pas une histoire, parce qu'y a rien donc je vais pas commencer à philosopher sur rien, raconter des choses sur rien. Il neige ces temps donc ça pourrait être une piste de ski parce que c'est blanc. C'est tout...

Dans le récit proposé à la dernière planche de l'épreuve du TAT, l'adolescent oscille entre échec d'une scénarisation interne ( « une je ne dis rien c'est pas une histoire, parce qu'y a rien ») et accrochage à l'actuel de l'expérience (« Il neige ces temps »), témoin du primat du perceptif.

\section{C - Charles (épreuve de Rorschach)}

- L'excitation initiée par la couleur et la dispersion du stimulus désorganise dans un premier temps la capacité de production de représentations (clivage entre la «multitude de choses » et « je ne distingue rien ») puis autorise la construction du stimulus (en appui sur la nomination de la couleur achromatique),

$\mathrm{X}: \mathrm{X}$ c'est 10 . Il y a une multitude de choses là-dessus... je ne distingue absolument rien là-dessus. Ah là peut-être ? Un crâne d'animal $(\mathrm{L}: 0$ :00:05) ? Là devant, la tache grise, on dirait l'avant d'un char Churchill, ouais, ça se tient.

On peut relever ici la fonction du renversement des planches comme soutien dans la production de la réponse, soutien conforté par la référence à une auto-représentation de la fonction-support (« ouais, ça se tient »).

\section{D - Charles (épreuve du TAT)}

- Avec le récit proposé à cette planche, l'inhibition perceptive témoigne de manière exemplaire des effets de blanc:

5. Il y en a combien là-dedans ? (prend la planche dans les mains)... Pff... je vois pas, là, je vois pas du tout, je suis carrément aveugle. (Clinicien : Vous avez pas une petite idée ?) Non, là je vois pas.

Axe de l'engagement transférentiel, témoin de la qualité des pare-excitations, ou axe du traitement de l'excitation dans le lien

Ce troisième axe met tout particulièrement en évidence les manifestations en forme d'agirs de transfert qui émaillent la production projective.

\section{A - Corneille (épreuve de Rorschach)}

- L'interrogation de l'intervention, de l'implication ou de l'intention du clinicien sur la production du stimulus s'inscrit sur un fond de déni de la dimension instituée du matériel, dans le contexte d'une fragilité de la représentation,

IV (enquête) : Le tout [au centre] c'est ça je peux pas en dire plus. Vous avez relevé les pieds ?)

Tout se passe comme si la marque du clinicien sur le stimulus se trouvait en mesure de garantir la fiabilité de l'expérience perceptive.

- Dans la même lignée, la sollicitation du clinicien sur le courant d'appartenance artistique du stimulus vient comme une tentative de réassurance dans un contexte de doute sur la production de la représentation (alors que la réponse proposée appartient aux réponses banales à cette planche) :

V : Axe de symétrie. Ça pourrait ressembler un peu à une chauve-souris. Ces images ça fait partie du moderne, de quelle époque ?

\section{D - Charles (épreuve du TAT)}

- L'interpellation explicite du clinicien à une sollicitation sensorielle contribue à soutenir le processus de mise en représentation :

13B : Vous avez pas plus concret à me montrer comme truc ? Genre quelque chose qui me saute à 
l'œil ? C'est l'histoire d'un gosse, c'est l'histoire d'un gosse qui a été abandonné par ses parents dans le désert et vit là-bas et se démerde tout seul et meurt dans cette cabane quand sera vieux enfin quand il est vieux (baille, s'agite, bouge les jambes).

On notera ici l'implication somatique et sensorimotrice de Charles dans la production de la réponse (traduction somatique de l'affect ?)

- Au retest, la mise en acte, sur le mode de la résolution dans l'ici et maintenant du vécu de carence, soutient lui aussi l'élaboration du récit :

13B' - retest : (il prend son portemonnaie et sort 2 billets de CHF 100, qu'il tient dans la main) C'est l'histoire d'un garçon qui attend son papa dans l'entrée de la maison (des pièces de monnaie tombent, il se baisse pour les ramasser) c'est tombé tout seul... heu... qui attend sont papa à l'entrée de la maison et il est en retard comme d'habitude et son papa il vient et il s'excuse et il lui offre un hochet et pis voilà tout le monde il est content.

— Enfin, la mise en question (la faillite ?) de la potentialité représentative s'exprime au risque de l'épuisement, avec un rabattement sur une approche strictement sensorielle :

16 (renverse la planche et la renverse à nouveau) : Oula... je vais voir beaucoup de neige blanc, voilà c'est fini. Ça m'inspire pas grand-chose je sais. Blanc. Et si vous deviez raconter une histoire ? Impossible avec un truc blanc, avec des contours blancs ça va être dur, ça va être difficile. C'était une question piège en fait. Vous m'avez épuisé avec toutes ces histoires qui ne me reste rien pour la dernière. Non rien d'autre que blanc, éventuellement blanc-jaune.

Au-delà de l'émergence persécutoire, on peut noter la désorganisation latente au cœur du discours, qui accompagne la perte de la différenciation forme/ fond (« Impossible avec un truc blanc, avec des contours blancs »).

\section{CONCLUSION}

Les différentes illustrations proposées montrent, de manière assez explicite, tout à la fois la richesse du panorama des catastrophes de symbolisation, considérées comme témoin du destin du traumatisme, et le caractère dynamique de celles-ci. Ces catastrophes de symbolisation, ou coups de théâtre dans la vie psychique, contiennent, en germe, des potentiels de réaménagement dont il s'agirait de suivre le destin dans leur émergence aux épreuves projectives, de planche en planche et d'une épreuve à l'autre ${ }^{3}$, au-delà de ce qui peut s'observer au sein même des exemples de réponses ou de récits isolés qui ont été présentés dans le cadre du présent article, dans une visée illustrative.

Cette reprise de la notion de catastrophe de symbolisation, qui s'inscrivait à l'origine, je le rappelle, dans une préoccupation pour une clinique psychosomatique, centrée ici sur une mise à l'épreuve d'une clinique de l'agir sexuel violent à l'adolescence, témoigne, s'il en était besoin, de la continuité des problématiques de l'agir (Roman, Dumet, 2009), de l'agir somatique (somatisation) à l'agir violent (auto- / hétéro adressé). Ces cliniques sont marquées, on le sait, par le jeu du clivage, qui porte sur des parts de l'expérience infantile écartées ou coupées de la subjectivité ; clinique du clivage du Moi, ou plus sûrement du clivage au Moi (Roussillon, 1991), qui rend compte d'un échec dans la symbolisation des expériences précoces, en deça du langage, éprouvées et non symbolisées (ou insuffisamment symbolisées).

La scène projective peut être une occasion de relance du travail de symbolisation, travail dont on sait bien qu'il n'emprunte pas des voies toutes linéaires : les catastrophes de symbolisation font partie de ces coups de théâtre de la vie psychique qui contribuent à témoigner et à réduire la part du clivé dans le jeu de la pulsion, de sa transformation et de son destin. À ce titre, c'est bien le trajet de la pulsion qui peut être évalué à partir du repérage des catastrophes de symbolisation, dans cette articulation et cette remise en jeu subtile entre traces perceptives, émergences affectives et constructions conceptuelles (Freud, 1896/1973), sur lesquelles les productions projectives, dans leur complexité expressive (verbales, textuelles, mimo-sensori-motrices...) peuvent utilement nous éclairer.

Enfin, on comprendra que ce repérage concerne, bien au-delà de la clinique des enfants ou des adolescents, également la clinique des adultes, dans le champ de ce qu'il est désormais habituel de nommer la clinique des souffrances ou pathologies narcissiques-identitaires (Roussillon, 1991, 2014). Cette clinique se révèle paradigmatique des catastrophes de symbolisation, en tant que celles-ci traduisent la précarité d'une élaboration d'expériences traumatiques précoces, inscrites, sur le mode de liaison non-symbolique, dans le temps d'avant le langage, comme en témoignent de précédents travaux en clinique adulte (Roman, 2014b).

3. La notion de fil projectif (Roman, 2015) permet de rendre compte de cette dynamique qui se déploie de planche en planche et d'épreuve en épreuve. 


\section{RÉFÉRENCES}

BAlier (Claude).- Psychanalyse des comportements sexuels violents, Paris, Presses universitaires de France, 1996.

BAlier (Claude).- La violence à la lumière des processus adolescents, Adolescence, XXXI, 1998, p. 117-126.

Brelet (Françoise).- Fantasme et situation projective, Paris, Dunod, 1986.

Ciavaldini (André).-Psychopathologie des agresseurs sexuels, Paris, Masson, 1999.

Ciavaldini (André).- L'agir : un affect inachevé, dans Boushira (J.), Parat (H.), L'affect, Paris, Presses universitaires de France, 2005, p. 137-162.

Dejours (Christophe).- L'inné et l'acquis. La cohabitation entre l'infantile (pulsionnel) et le pubertaire (instinctuel), Carnet psy, VI, 173, 2013, p. 37-41.

Freud (Sigmund).- Lettre du 6 décembre 1896 à W. Fliess [1896], dans Freud (S.), La naissance de la psychanalyse, Paris, Presses universitaires de France, 1973, p. $153-154$.

FREUd (Sigmund).- Remarques sur l'amour de transfert [1915], dans CEuvres complètes XII, Paris, Presses universitaires de France, 2005, p. 197-211.

Gutton (Philippe).- Le pubertaire, Paris, Presses universitaires de France, 1991.

Gutton (Philippe).-Adolescens, Paris, Presses universitaires de France, 1996.

JANIN (Claude).- Figures et destins du traumatisme, Paris, Presses universitaires de France, 1996.

RAVIT (Magali).- Du traumatisme à la fascination dans la clinique du passage à l'acte, Psychologie clinique et projective, XVI, 2010, p. 29-49.

Ravit (Magali), Di Roco (Vincent), Bécache (Ève), CARKa (Delphine).- De l'illusion meurtrie à la désillusion meurtrière, Psychologie clinique et projective, XVIII, 2013, p. 167-184.

Roman (Pascal).- Troubles somatiques et catastrophes de symbolisation, Psychologie clinique et projective, III,
1, 1997a, p. $75-87$.

Roman (Pascal).- La méthode projective comme dispositif à symboliser, dans Roman (P.) et coll., Projection et symbolisation chez l'enfant, Presses universitaires de Lyon, 1997b, p. 37-51.

Roman (Pascal).- Les violences sexuelles à l'adolescence. Comprendre, accueillir, prévenir, Paris, Elsevier-Masson, 2012.

Roman (Pascal).- Les violences sexuelles dans la famille à l'adolescence et l'épreuve de la différenciation [2014a], dans Houssier (F.), Violences dans les liens familiaux, Paris, In Press, 2014, p. 69-84.

Roman (Pascal).- Violence et psychopathologie : l'agir sexuel violent et la butée de la perversion - Contribution des épreuves projectives [2014b], dans Gaillard (B.), La psychologie criminologique ( $2^{\mathrm{e}}$ éd.), Paris, In Press, 2014, p. 99-124.

Roman (Pascal).-Le Rorschach en clinique de l'enfant et de l'adolescent (2 ${ }^{\mathrm{e}}$ éd.), Paris, Dunod, 2015.

Roman (Pascal), Dumet (Nathalie).- Des corps en actes : symbolisation/désymbolisation à l'adolescence, Cliniques méditerranéennes, LXXIX, 2009, p. 207-227.

Roussillon (René).- Paradoxe et situations limites de la psychanalyse, Paris, Presses universitaires de France, 1991.

Roussillon (René).- L'homosexualité primaire et le partage de l'affect, dans Mellier (D.), Vie émotionnelle et souffrance du bébé, Paris, Dunod, 2002, p. 73-93.

Roussillon (René) et coll.- Manuel de psychologie et de psychopathologie clinique générale, Paris, ElsevierMasson (2éd.), 2014.

Virole (Benoît).- Morphogénèse des stéréotypies motrices dans l'autisme infantile, Sémiotiques, 3, 1992, p. 31-62.

WinnicotT (Donald-Wood).- $\quad$ La crainte de l'effondrement [1989], dans Winnicott (D.-W.), La crainte de l'effondrement et autres textes, Paris, Gallimard, 2000, p. 205-216. 